\title{
Discrepancy between Motor and Cognitive Control in Adults with Intellectual Disabilities
}

\author{
Shogo Hirata ${ }^{1, *}$, Hideyuki Okuzumi ${ }^{2}$, Yoshio Kitajima $^{1}$, Tomio Hosobuchi $^{3}$ and \\ Mitsuru Kokubun ${ }^{2}$
}

${ }^{1}$ Faculty of Education, Chiba University, Japan

${ }^{2}$ Faculty of Education, Tokyo Gakugei University, Japan

${ }^{3}$ Faculty of Education, Saitama University, Japan

\begin{abstract}
Purpose: To investigate the relationship between motor and cognitive control in adults with intellectual disabilities (ID), focusing on two aspects, speed and accuracy.

Method: Participants were 62 adults with ID aged 20 to 47 years. Their intelligence quotients (IQ) ranged from 13 to 61 . Nine of the adults with ID had Down syndrome, and 8 of the adults had autism. We conducted three tasks: seal affixation task, tray-carrying task, and the Matching Familiar Figures Test (MFFT). The seal affixation and tray-carrying tasks are motor tasks we devised that can separately measure the speed and accuracy of motor control. MFFT is a cognitive control task that can be used to evaluate cognitive styles, such as impulsive-reflective.

Results: Adults with ID showed high motor accuracy and similar motor speed regardless of their MFFT performance. That is, discrepancies between motor and cognitive control existed in adults with ID.

Conclusions: The results of this study indicate that some types of motor control problem may become unclear with growth. A longitudinal investigation focused on the motor skill development of persons with ID is therefore necessary.
\end{abstract}

Keywords: Motor control, cognitive control, intellectual disability, speed and accuracy.

Intellectual disabilities (ID) refer to intellectual and adaptive difficulties that have their onset before 18 years of age [1]. The core features of these disabilities are general intellectual or cognitive deficits. However, it is also well-known that children and adults with ID have poor motor skills. Many empirical studies have revealed the existence of motor skill impairments in persons with ID. For instance, they show lower performance in standardized motor assessments such as the BurinksOseretsky test of motor proficiency or the Movement Assessment Battery for Children, and have weaker fine motor skills than gross motor skills [2-4].

In the study of motor skill impairments of persons with ID, it is very important to define which viewpoint is being used to evaluate motor control. In general, 'slowness' of movement seems to be one of the major characteristics of motor control in persons with ID, especially those with Down syndrome (DS) [3, 5, 6]. However, several researchers have suggested the possibility that slow movement speed is a product of a control strategy to guarantee movement accuracy or safety in some children with ID [6, 7]. That is to say, motor ability of some children with ID is not lower because of motor slowness. Thus, when the motor

*Address correspondence to this author at the Faculty of Education, Chiba University, 1-33 Yayoi, Inage, Chiba, 263-8522, Japan; Tel: +81(042)-3297385; Fax: +81(042)-329-7385; E-mail: r093002g@st.u-gakugei.ac.jp control of children with ID is evaluated, not only motor speed but also motor accuracy is important. Based on this, Hirata et al. [8] investigated the speed and accuracy of fine motor control in children with ID, and attempted to classify the features of their motor control. They were able to classify children with ID into four groups: fast and accurate, fast and inaccurate (speed domination), slow and accurate (accuracy domination), and slow and inaccurate. Thus, it is considered that the features of motor control in children with ID are not homogeneous, but have distinct groups.

In their most recent study, Hirata et al. [9] have taken some important steps toward understanding the motor-cognitive control link in children with ID. They drew attention to the similarity between features of fine motor control in children with ID and features of some types of cognitive control, such as 'impulsivityreflection'. In the field of developmental psychology, impulsivity and reflection are well-known and studied. According to these concepts, children who respond more rapidly and tend to make more errors are 'impulsive', while those who respond relatively slowly and tend to make fewer errors in problem-solving situations or cognitive control tasks are 'reflective' [10, 11]. Hirata et al. [9] considered 'impulsive' to correspond to the 'fast and inaccurate' group of children with ID, and 'reflective' to the 'slow and accurate'. To test this hypothesis, they investigated the 
relationship between motor and cognitive control of children with ID. The 3 tasks conducted by Hirata et al. [9] were a seal affixation task [8], a tray-carrying task [7], and the Matching Familiar Figures Test (MFFT). The seal affixation task and tray-carrying task are motor control tasks for measuring the speed and accuracy of motor controls. The seal affixation task is a fine motor task, whereas the tray-carrying task is a gross motor task. The MFFT is the most widely used cognitive control task for measuring impulsivity and reflection [10]. The tasks in this test require subjects to search through a number of similar pictures to find one that matches a criterion picture exactly. The measurement method in the MFFT is widely accepted and used in current personality psychology and developmental psychology (see for example, [11]). Hirata et al. [9] classified children with ID into four groups based on their performance on MFFT: fast and accurate, impulsive (fast and inaccurate), reflective (slow and accurate), and slow and inaccurate; they then investigated the motor performance of each group. As a result, it was found that the motor performance of each group corresponded to their characteristics seen in MFFT, i.e., the impulsive group took less time to complete two motor control tasks and their motor accuracies were low. In contrast, the reflective group took more time and their motor accuracies were high. The performance of the other two groups also corresponded to their characteristics seen in MFFT. These results suggest the possibility that the motor control features of children with ID correspond to their cognitive control features. That is, a motor-cognitive control link existed in the children with ID. Although the above finding by Hirata et al. [9] is very interesting, no further research has been conducted using this approach.

In Hirata et al.'s study [9], their participants were 39 children in the developmental period aged 8-15 years. Is there a similar relationship between motor and cognitive control in adults with ID? In recent years, neuroimaging and neuropsychology studies have revealed that cognitive control or executive control processes depend not only on prefrontal areas such as the dorsolateral prefrontal cortex, but also on motorrelated areas such as the premotor cortex, supplementary motor area and cerebellum [12, 13]. The fact that motor control and cognitive control have common brain mechanisms is considered to be one reason for the existence of the motor-cognitive control link in children with ID. Moreover, several researchers have found that performance in motor control tasks and tasks that evaluated executive function or cognitive control were linked in non-handicapped children [11, $14,15]$. Thus, it is possible that a similar relationship exists in adults with ID. An investigation addressing this point may provide further understanding and new insights into the psychological characteristics of adults with ID. The present study will attempt to clarify this point.

To summarize, the purpose of this study was to investigate the relationship between motor and cognitive control in adults with intellectual disabilities (ID) by using the same tasks as Hirata et al. [9].

\section{METHOD}

\section{Participants}

The participants were 62 adults with ID free from sensory and motor impairments such as blindness, low vision, deafness, and cerebral palsy. Chronological age (CA) ranged from 20 to 47 years (median $(\mathrm{Me})=34.0$, quartile deviation $(Q)=4.0$ ), and intelligence quotients (IQ) ranged from 13 to $61(\mathrm{Me}=29.5, \mathrm{Q}=6.5)$. Among the 62 participants with ID, 9 were adults with Down syndrome ( 3 males, 6 females), 8 were adults with autism (8 males), and 45 were adults without either syndrome (28 males, 17 females). Down syndrome and autism were diagnosed by medical doctors, and there were no participants with both Down syndrome and autism. Descriptive statistics for CA and IQ for all three groups (Down syndrome, autism and others) can be seen in Table 1. The Kruskal-Wallis test was conducted to analyze the differences in $C A$ and $I Q$ among the three groups. The differences were not significant for either CA or IQ (CA: $\chi^{2}(2)=3.86, p>.05 ; \quad I Q: \chi^{2}$ $(2)=2.76, p>.05)$. Participants' IQs were measured with the Tanaka-Binet Intelligence scale, which is a standardized and commonly-used test in Japan that has been sufficiently validated against the Wechsler scale. The Tanaka-Binet scale is suitable for assessing the intelligence of persons with ID because it is applicable to a wider range of IQ than the Wechsler test.

Table 1: Descriptive Statistics for Each Clinical Type

\begin{tabular}{|c|c|c|c|c|c|}
\hline \multirow{2}{*}{} & & \multicolumn{2}{|c|}{ CA } & \multicolumn{2}{c|}{ IQ } \\
\cline { 2 - 6 } & $\mathbf{n}$ & Me & $\mathbf{Q}$ & Me & Q \\
\hline \hline Down syndrome & 9 & 34.0 & 2.0 & 25.0 & 4.0 \\
\hline Autism & 8 & 27.5 & 3.9 & 26.5 & 5.5 \\
\hline Other & 45 & 34.0 & 3.5 & 31.0 & 6.5 \\
\hline
\end{tabular}




\section{Tasks}

\section{Seal Affixation Task [8]}

The seal affixation task is a motor control task designed to separately measure the speed and accuracy of fine motor control. In this study, a circular frame $20 \mathrm{~mm}$ in diameter was drawn on an A4 sheet of paper. A seal $20 \mathrm{~mm}$ in diameter was presented in the start position, and the time needed (sec) to affix the seal to a circular frame $150 \mathrm{~mm}$ away was measured. Participants were asked to affix the seal to the circular frame as quickly as possible, but to keep it within the frame. The seal was pre-stuck to an experimenter's index finger and presented to the participant. Measurement began at the moment the participant took the seal from the experimenter's finger with his or her dominant hand, and the measurement ended at the moment the participant's finger came off the seal. In this study, hand dominance was determined by asking each participant a question: 'Which hand do you use to hold the chopsticks when you eat rice?' The experimenter measured the time with a stop-watch. Three trials were conducted for measurements. The test paper was scanned into the computer, and then the gap $(\mathrm{mm})$ between the center of the circular frame and the center of the affixed seal was measured. The average time score and gap score for three trials were used as representative values for each participant.

\section{Tray-Carrying Task [7]}

The tray-carrying task is a motor control task designed to separately measure the speed and accuracy of gross motor control. For the tray-carrying task, participants were given a tray $(26 \mathrm{~cm}$ in a diameter) by the experimenter at the start line with a glass $(9 \mathrm{~cm}$ in height, with a diameter of $7 \mathrm{~cm}$ at the top, $4.5 \mathrm{~cm}$ at the bottom, and a capacity of $225 \mathrm{ml}$ ) holding $210 \mathrm{ml}$, and instructed to carry it to a goal line 3 $\mathrm{m}$ away as fast as they could without spilling any water. The time to reach the goal line was measured with a stop-watch. The amount of water remaining in the glass on completion of the task was measured with a measuring cylinder, and this value was subtracted from $210(\mathrm{ml})$ to calculate the amount of water spilled. The averages of two trials were used as representative participant variables.

\section{Matching Familiar Figures Test (MFFT)}

Hirata et al. [9] used three items in the MFFT for preschool children developed earlier by Wright [16]. Only three items were used in order to reduce the participants' burden as much as possible. Each item consisted of a model drawing and 6 or 5 highly very similar drawings (two 6-drawing versions and one 5drawing version were used), one of which was identical to the model drawing. The participant had to point out the picture that was identical to the model drawing. If mistaken, the participant tried again up to 3 times. Performance was assessed from the time taken to give the first response (response latency, sec) and the total number of errors. The experimenter measured the time with a stop-watch. The sum of the response latencies and the number of errors were used as representative values for each participant. Before conducting MFFT, 'distinction of an animal' task, which is part of the Tanaka-Binet Intelligence scale for the 2-year-old class used to evaluate fundamental visual matching ability, was conducted. Only those who passed this task carried out MFFT. Hirata et al. [9] used data for 5-yearold children without disabilities to classify the children with ID, because, looking at the developmental change of the performance of MFFT administered according to the same procedure as children with ID, the total errors become nearly zero and the response latency became faster after 5 years of age [17]. This study also classified MFFT performance into groups by using the same criteria. The participants whose response latency was greater than the median for 5-year-old children $(>14 \mathrm{sec})$ and who had higher total errors than the median for 5 -year-olds $(>1)$ were placed in the 'slowinaccurate' category. The 'impulsive (fast-inaccurate)' group consisted of participants whose response latency was less than $14 \mathrm{sec}$ but had higher total errors (>1). The 'reflective (slow-accurate)' group consisted of participants whose response latency was greater than 14, but had low total errors. The 'fast-accurate' group consisted of participants whose response latency was less than 14 , but had low total errors.

\section{Procedure}

Ethical approval for the study was obtained from the Research Ethics Board at Tokyo Gakugei University. All participants were users in two institutions. Consent to participate in the study by adults with intellectual disabilities was obtained from the institutions where they lived, and participants were under no obligation to take part in the tests. The purpose of the tests was explained, and only persons who freely and voluntarily consented to participate were included. We tried to measure performance of all users without sensory and motor impairments in each institution, but did not measure the performance of users who had a clear difficulty in understanding instructions. Each participant was assessed in two sessions. Two motor tasks and 
Table 2: Descriptive Statistics of the Motor and Cognitive Control Tasks $(n=62)$

\begin{tabular}{|c|c|c|c|}
\hline & Me & $\mathbf{Q}$ & Range \\
\hline \multicolumn{4}{|l|}{ Seal affixation task } \\
\hline Time (sec) & 4.5 & 1.5 & $1.9-21.7$ \\
\hline Gaps (mm) & 0.9 & 0.2 & $0.4-1.9$ \\
\hline \multicolumn{4}{|l|}{ Tray-carrying task } \\
\hline Time (sec) & 5.7 & 1.3 & $3.0-13.7$ \\
\hline Water spilled (ml) & 0.0 & 0.5 & $0.0-14.0$ \\
\hline \multicolumn{4}{|l|}{ MFFT } \\
\hline Response latency (sec) & 14.5 & 7.0 & $3.0-119.0$ \\
\hline Total error & 2.0 & 1.9 & $0.0-9.0$ \\
\hline
\end{tabular}

the MFFT were administered to the participant in the first session, and the Tanaka-Binet intelligence scale in the second. These sessions were conducted in a private room in each institution. The Tanaka-Binet intelligence scale was conducted on another day. The participant's CA and clinical types were surveyed from the personal histories kept at institutions.

\section{Statistical Analysis}

SPSS (ver. 12.0) was used for statistical analysis. A significance level of $p<0.05$ was used for all analyses. All the measured values (i.e., seal time, seal gaps, tray time, water spilled, response latency, and total error; see Table 2) were tested for deviation from normality. The tests revealed that not all the measures were normally distributed. Thus, nonparametric analyses were used in this study. The Kruskal-Wallis test was used to analyze the medians of each group. When differences between groups were significant, the data were subjected to post hoc analysis by Dunn's test. The chi-square test was used to analyze the relationship between the cognitive style and clinical types of ID.

\section{RESULTS}

\section{Performance of Motor Control Tasks in Each Group}

Table 2 presents the descriptive statistics for all measured variables used in this study. In order to clarify the link between motor and cognitive control, we classified the performance of MFFT into four groups, and then compared the motor task performance and various attributes of each group. Table 3 shows the medians (Me) and quartile deviation ( $Q$ ) of the motor and cognitive control task for each group. Time taken to complete each motor task of the reflective group was longest in adults with ID. Each motor time of the other three groups was not so different. The seal gap of each group was very small ( $1 \mathrm{~mm}$ or less), and the amount of water spilled was 0 in all groups. The Kruskal-Wallis test was conducted to analyze the difference in performances of motor control tasks among the four groups. The main effect of grouping was significant only when the time of the motor control task was a dependent variable (Seal time: $\chi^{2}(3)=12.1, p<.05$; Seal gap: $\chi^{2}(3)=3.8, p>.05$; Tray time: $\chi^{2}(3)=11.5$, $p<.05$; water spilled; $\left.\chi^{2}(3)=0.5, p>.05\right)$. Post hoc test showed that the seal affixation time of the reflective group was significantly longer than that of three residual groups, and the tray carrying time of the reflective group was significantly longer than that of the fast-accurate group.

Table 3: Medians and Quartile Deviations of Cognitive and Motor Task Performance for Each Group

\begin{tabular}{|c|c|c|c|c|c|c|c|c|}
\hline & \multicolumn{2}{|c|}{$\begin{array}{l}\text { Slow-inaccurate } \\
\qquad(n=11)\end{array}$} & \multicolumn{2}{|c|}{$\begin{array}{l}\text { Impulsive } \\
\quad(n=22)\end{array}$} & \multicolumn{2}{|c|}{$\begin{array}{l}\text { Reflective } \\
\quad(n=16)\end{array}$} & \multicolumn{2}{|c|}{$\begin{array}{l}\text { Fast-accurate } \\
\qquad(n=13)\end{array}$} \\
\hline & Me & $\mathbf{Q}$ & Me & $\mathbf{Q}$ & Me & $\mathbf{Q}$ & Me & $\mathbf{Q}$ \\
\hline \multicolumn{9}{|l|}{ MFFT } \\
\hline Response latency (sec) & 26.0 & 5.8 & 9.5 & 3.0 & 30.5 & 12.4 & 13.0 & 2.5 \\
\hline Total errors & 2.0 & 1.5 & 5.0 & 1.5 & 0.0 & 0.5 & 1.0 & 0.5 \\
\hline \multicolumn{9}{|l|}{ Seal affixation task } \\
\hline Time (sec) & 4.1 & 0.7 & 4.1 & 1.0 & 7.3 & 1.7 & 4.7 & 0.9 \\
\hline Gaps (mm) & 0.8 & 0.1 & 0.9 & 0.2 & 0.9 & 0.2 & 0.7 & 0.1 \\
\hline \multicolumn{9}{|l|}{ Tray-carrying task } \\
\hline Time (sec) & 5.1 & 1.6 & 5.7 & 0.9 & 6.8 & 1.5 & 4.3 & 1.0 \\
\hline Water spilled (ml) & 0.0 & 0.8 & 0.0 & 0.9 & 0.0 & 0.5 & 0.0 & 0.5 \\
\hline
\end{tabular}


Table 4: Descriptive Statistics for Each Group

\begin{tabular}{|c|c|c|c|c|c|c|c|c|c|}
\hline & & \multicolumn{2}{|c|}{ Slow-inaccurate } & \multicolumn{2}{|c|}{ Impulsive } & \multicolumn{2}{|c|}{ Reflective } & \multicolumn{2}{|c|}{ Fast-accurate } \\
\hline \multirow[t]{7}{*}{ Number } & Down syndrome & \multicolumn{2}{|c|}{5} & \multicolumn{2}{|c|}{0} & \multicolumn{2}{|c|}{4} & \multicolumn{2}{|c|}{0} \\
\hline & Autism & \multicolumn{2}{|c|}{0} & \multicolumn{2}{|c|}{5} & \multicolumn{2}{|c|}{1} & \multicolumn{2}{|c|}{2} \\
\hline & Other & \multicolumn{2}{|c|}{6} & \multicolumn{2}{|c|}{17} & \multicolumn{2}{|c|}{11} & \multicolumn{2}{|c|}{11} \\
\hline & & \multicolumn{2}{|c|}{ Slow-inaccurate } & \multicolumn{2}{|c|}{ Impulsive } & \multicolumn{2}{|c|}{ Reflective } & \multicolumn{2}{|c|}{ Fast-accurate } \\
\hline & & Me & $\mathbf{Q}$ & Me & $\mathbf{Q}$ & Me & $\mathbf{Q}$ & Me & $\mathbf{Q}$ \\
\hline & $\mathrm{CA}$ & 34.0 & 3.0 & 33.0 & 3.4 & 34.0 & 5.0 & 37.0 & 3.0 \\
\hline & IQ & 25.0 & 6.3 & 27.0 & 5.0 & 31.0 & 5.9 & 36.0 & 2.0 \\
\hline
\end{tabular}

\section{Attributes of Each Group in Adults with ID}

Next, Table 4 shows descriptive values of the various attributes of each group. Adults with Down syndrome are included in two groups: slow-inaccurate and reflective. The chi-square test revealed that there are many adults with Down syndrome in the slowinaccurate group $\left(\chi^{2}(6)=18.1, p<.05\right)$. Adults with autism tended to be in the impulsive group. There were no large differences among the groups in their CA $\left(\chi^{2}\right.$ $(3)=2.26, p>.05)$ and $I Q\left(\chi^{2}(3)=7.66, p>.05\right)$.

\section{DISCUSSION}

The purpose of this study was to investigate the relationship between motor and cognitive control in adults with ID from the speed and accuracy of their behavior. In adults with ID in this study, only the motor performance of the reflective group corresponded to their characteristics seen in MFFT, i.e., they perform slowly but accurately in motor and cognitive control tasks. In general, however, it could be said that adults with ID showed an almost high motor accuracy and similar motor speed without depending on their performance on MFFT. That is, discrepancies between motor and cognitive control existed in adults with ID, unlike children with ID [9]. As mentioned in the Introduction, motor control features of children with ID correspond to their cognitive control features. Naturally enough, one possible reason for this difference between children and adult with ID is in their chronological age.

In this study, motor accuracies of the impulsive group and slow-inaccurate group were higher than the value expected by total error of MFFT. And, motor speed of the slow-inaccurate group was also faster than the value expected by response latency of MFFT. In brief, the features of motor control of the impulsive and slow-inaccurate groups are similar to those of the fast-accurate group. It is considered that such motorskillulness which cannot be explained from the cognitive control features of these groups may be a reflection of past experience and learning. In current cognitive neuroscience, MFFT is considered to be a cognitive control task that requires response inhibition and planning [18, 19]. Hirata et al. [9] suggest the possibility that the features of motor and cognitive control tasks in impulsive children with ID are related to inhibitory dysfunction, and that the features of slowinaccurate children are related to planning deficit. Because the performance of the MFFT could be classified into four groups (in the same way as a child) in adults with ID, it is considered that there is the same cognitive deficit to be identified in impulsive and slowinaccurate groups of adults with ID. Results of this study indicate that these cognitive deficits do not strongly influence the performances of simple motor control tasks such as seal affixation and tray-carrying task. In daily life, persons with ID study in school and work in society using various hand tools and carrying various objects. Such longtime experience may develop motor skillfulness in some types of adults with ID. There are few studies about the positive consequence of aging in adults with ID. We consider that there is a particular need for a longitudinal investigation on the development of motor control in persons with ID. However, it should be noted that we do not insist that motor control of adults with ID become completely intact. Many authors agree that complex motor control involves complex cognitive processes such as shifting, inhibition and monitoring (i.e., [20]). The motor control tasks conducted in this study can be performed by healthy 3-year-old children $[7,8]$. A different result may have been obtained if we had conducted a more difficult motor control task for adults with ID. That is, cognitive control deficits of adults with ID may be related with performances of 
such motor control task. On the other hand, the reason why the motor control speed of the reflective group corresponded to their characteristics seen in MFFT is not yet clear. In Hirata et al. [9], the features of motor and cognitive control tasks in reflective children with ID were related to control strategy to guarantee accuracy. Is such a strategy difficult to revise once it is acquired? We will address these issues in a future article.

$\mathrm{CA}, \mathrm{IQ}$, the existence of Down syndrome, and the existence of autism were not decisive factors that divided the four groups of children with ID. CA was almost equal and there was no clear difference between groups in IQ. Although there were many children with Down syndrome in the slow-inaccurate group, there were also many in the reflective group. Slowness of cognitive speed is common in these cognitive styles, and such features may be characteristic of children with Down syndrome. On the other hand, the most common cognitive style in the adults with autism was impulsive. However, two autistic adults were included in the fast-accurate group and an autistic adult in the reflective group. Therefore, it is considered that a particular 'impulsive - reflective' aspect of cognitive style does not exist in autism. These four factors are the most fundamental attributes of adults with ID. It is interesting that these factors do not have a really strong influence on individual differences in the speed and accuracy of cognitive control. In future study, we will try to check this hypothesis.

\section{LIMITATIONS}

As with Hirata et al. [9], the number of participants with Down syndrome and autism in this study was not large enough to make a robust conclusion. Moreover, to secure the validity of each cognitive group, a wider array of cognitive control tasks should be used to group the adults with ID. It will also be necessary to examine the speed and accuracy of motor and cognitive control in non-handicapped adults with normal IQ.

\section{ACKNOWLEDGEMENTS}

The authors would like to thank the participants who made this work possible. This study was supported by a Grant-in-Aid for Scientific Research (245482) to Hirata from the Japan Society for the Promotion of Science.

\section{REFERENCES}

[1] American Psychiatric Association. Diagnostic and statistical manual of mental disorders (4th ed.). Washington, DC: American Psychiatric Association 2000.
[2] Connolly BH, Michael BT. Performance of retarded children, with and without Down syndrome, on the Bruininks Oseretsky test of motor proficiency. Phys Ther 1986; 66: 344-348.

[3] Wuang YP, Wang CC, Huang MH, Su CY. Profiles of cognitive predictors of motor functions among early schoolage children with mild intellectual disabilities. J Intellect Disabil Res 2008; 52: 1048-1060.

http://dx.doi.org/10.1111/j.1365-2788.2008.01096.x

[4] Vuijk PJ, Hartman E, Scherder E, Visscher C. Motor performance of children with intellectual disability and borderline intellectual functioning. J Intellect Disabil Res 2010; 54: 955-965

http://dx.doi.org/10.1111/j.1365-2788.2010.01318.x

[5] Ushiyama M, Hosokawa T, Okuzumi H, Tanaka A. Aimed movement control in individuals with mental retardation. Japan J Spec Educ 2002; 39: 115-122.

[6] Latash ML. Neurophysiological Basis of Movement. Second Edition. Champaign: Human Kinetics 2008.

[7] Kokubun M. Are children with Down syndrome less careful in performing a tray-carrying task than children with other types of mental retardation? Percept Mot Skills 1999; 88: 11731176.

http://dx.doi.org/10.2466/pms.1999.88.3c.1173

[8] Hirata S, Okuzumi H, Kitajima Y Hosobuchi T, Kokubun M. Speed and accuracy of motor actions in persons with intellectual disabilities. J Educ Res 2010; 21: 58-70. (in Japanese with English abstract).

[9] Hirata S, Okuzumi H, Kitajima Y Hosobuchi T, Kokubun M. Speed and accuracy of motor and cognitive control in children with intellectual disabilities. Int J Dev Disabil 2013; 59; $166-178$.

http://dx.doi.org/10.1179/2047387712Y.0000000010

[10] Kagan J, Rosman BL, Day D, Albert J, Philips W. Information processing in the child: Significance of analytic and reflective attitudes. Psychol Mono 1964; 78: 1-37. http://dx.doi.org/10.1037/h0093830

[11] Rosey F, Keller J, Golomer E. Impulsive-reflective attitude, behavioural I nhibition and motor skills: Are they linked? Int J Behav Dev 2010; 34: 511-520. http://dx.doi.org/10.1177/0165025409361009

[12] Diamond A. Close interrelation of motor development and cognitive development and of the cerebellum and prefrontal cortex. Child Dev 2000; 71: 44-56.

http://dx.doi.org/10.1111/1467-8624.00117

[13] Davis EE, Pitchford NJ, Jaspan T, McArthur D, Walker D. Development of cognitive and motor function following cerebellar tumour injury sustained in early childhood. Cortex 2010; 46: 919-932.

http://dx.doi.org/10.1016/j.cortex.2009.10.001

[14] Wassenberg R, Feron FJ, Kessels AG, et al. Relation between cognitive and motor performance in 5- to 6-year-old children: results from a large-scale cross-sectional study. Child Dev 2005; 76: 1092-1103.

http://dx.doi.org/10.1111/j.1467-8624.2005.00899.x

[15] Livesey D, Keen J, Rouse J, White F. The relationship between measures of executive function, motor performance and externalising behaviour in 5- and 6-year-old children. Hum Mov Sci 2006; 25: 50-64. http://dx.doi.org/10.1016/j.humov.2005.10.008

[16] Wright JC. The Kansas reflection-impulsivity scale for preschoolers. Cemrel, Inc. 1971.

[17] Hirata S, Okuzumi H, Kitajima Y, Hosobuchi T, Kokubun M. Impulsivity - Reflection in persons with intellectual disabilities. J Educ Res 2011; 25: 99-107. (in Japanese with English abstract).

[18] Das, JP. Planning: Theoretical Considerations and Empirical Evidence. Psychol Res 1980; 41: 141-151. http://dx.doi.org/10.1007/BF00308652 
[19] Johnston K, Madden AK, Bramham J, Russel A. Response inhibition in adults with autism spectrum disorder compared to attention deficit / hyperactivity disorder. J Autism Dev Disord 2011; 41: 903-912.

http://dx.doi.org/10.1007/s10803-010-1113-9
[20] Sergent J. The cognitive-energetic model: an empirical approach to attention-deficit hyperactivity disorder. Neurosci Behav Rev 2000; 24: 7-12.

http://dx.doi.org/10.1016/S0149-7634(99)00060-3 